\title{
Characterization and burden of severe eosinophilic asthma in New Zealand: Results from the HealthStat Database
}

\author{
Sumitra Shantakumar, ${ }^{1}$ Yu-Fan Ho, ${ }^{1}$ Janine Beale, ${ }^{2}$ Barry Gribben ${ }^{3}$ \\ ${ }^{1}$ Department of Real World Evidence and Epidemiology, GlaxoSmithKline, GSK Asia House, Singapore \\ ${ }^{2}$ Department of Medical Affairs, GlaxoSmithKline, GSK Auckland, New Zealand \\ ${ }^{3}$ CBG Health Research, Auckland, New Zealand
}

\begin{abstract}
Background: This retrospective cohort study aimed to characterize epidemiology, medication use and healthcare resource utilization (HCRU) of patients diagnosed with severe eosinophilic asthma (SEA) compared to other patients with asthma in New Zealand.

Methods: Adult patients with asthma with no concurrent diagnosis of Chronic Obstructive Lung Disease (COPD) were identified from the HealthStat primary care database and the National Minimum Dataset using asthma diagnosis, hospital codes and prescriptions. Patients with SEA were identified using a 1-year baseline period (2011) and were those with: inhaled corticosteroid prescription above medium dose (including high dose) plus controller medication, $\geq 2$ exacerbations, and eosinophils $\geq 300$ cells $/ \mu 1$ (or $\geq 150$ in 6 weeks prior to index date); patients were followed for 1 year (2012).

Results: 160/3,276 (4.9\%) asthmatics with available eosinophil counts met SEA criteria. Patients with SEA were more likely to be Māori, former smokers, have more comorbidities, higher mean BMI and higher neutrophil counts compared with other patients with asthma. In the follow up period, SEA patients had over 4 times as many exacerbations; incidence of exacerbations of the same frequency was highest in Māori patients.

Conclusions: Compared with other patients with asthma, SEA patients had over 1.5 times as many respiratory treatment prescriptions and higher all-cause HCRU and total healthcare costs; asthma-related healthcare costs were 3.6 times greater.
\end{abstract}

Key words: Severe eosinophilic asthma; New Zealand; epidemiology; costs.

Correspondence: Barry Gribben, CBG Health Research, PO Box 45173, Te Atatu Peninsula, Auckland, 0651, New Zealand. E-mail: barry.gribben@cbg.co.nz

Contributions: SS, Y-FH, JB and BG contributed to the conception, design, execution or analysis, and interpretation of the data. All authors approved the final version to be published after critically revising the manuscript/publication for important intellectual content.

Conflict of interest: This study was funded and conducted by GSK (GSK study number PRJ2407/208970). SS and Y-FH are employees of GSK and hold stocks/shares in GSK. JB is an employee of GSK but does not hold stock/shares in GSK. BG is a shareholder in HealthStat Ltd. that received funding to conduct the study. The authors report no other conflicts of interest in this work.

Funding: This study was sponsored by GSK (PRJ2407/208970).

Availability of data and materials: The dataset supporting the conclusions of this article is available in the HealthStat primary care repository.

Ethics approval and consent to participate: This study is an extension of an existing retrospective observational study of asthma patients that was approved by the relevant institutional review board in New Zealand, the Health and Disability Ethics Committee. All data for the present study other than the eosinophil counts had been collected for the previous asthma study in the same HealthStat patient population. For the present study, the GPs included in the HealthStat database were re-contacted to obtain permission to receive routine laboratory data with complete blood count test results for all patients. Prior to contacting the GPs, an amended ethics approval was obtained from the Health and Disability Ethics Committee on $1^{\text {st }}$ August, 2016 (ethics reference number 13/CEN/139/AM03).

Consent for publication: Not applicable. 


\section{Introduction}

Patients with severe asthma (SA) are a high-risk population who suffer from continuing symptoms and acute exacerbations. Those with severe disease have a significant unmet need in terms of effective treatment, and therapies to target specific severe asthma phenotypes are needed. In some patients, SA is associated with persistent eosinophilic airway inflammation (severe eosinophilic asthma; SEA), and disease that remains poorly controlled despite continuous treatment with high-dose inhaled glucocorticoids (ICS). In these patients with SEA, high blood eosinophil levels may be associated with frequent asthma exacerbations, which are a major clinical concern and create a significant burden on the patient and the healthcare system [1].

Estimates suggest that patients with SA represent approximately $5-10 \%$ of the total asthma population [2]; however the treatment of frequent, acute exacerbations contributes to a disproportionately high level of healthcare resource utilization (HCRU) i.e., $\sim 50 \%$ by this patient group $[3,4]$. Health-related quality of life is also severely impaired for SA patients and their caregivers [5-7] and disease severity is associated with societal costs through loss of work productivity and absenteeism [8].

Medicated asthma prevalence in New Zealand is high and was reported at $14.1 \%$ for children and $11.1 \%$ for adults in $2011-2013$ [9]. Moreover, in 2001-2003 the International Study of Asthma and Allergies in Children noted the prevalence of severe asthma symptoms in children in New Zealand to be one of the highest in the world (>7.5\%) [10]. Ethnicity-specific differences in disease have been described in New Zealand, whereby Māori have been shown to be more symptomatic than non-Polynesians (ethnicities other than Māori or Pacific Islanders), and asthma symptoms in Māori increase with age compared with non-Māori [11]. Those of Māori and Pacific ethnicities are also more likely to be hospitalised due to asthma and have higher asthma-related mortality rates [9], and Māori and Pacific children are more likely to experience severe asthma symptoms [12,13], utilise oral corticosteroid (OCS) treatment, and have asthma-related hospitalisations [14].

Despite the substantial mortality and morbidity burden associated with SA there are currently few studies describing the prevalence of SA phenotypes, including SEA, in the AsiaPacific/Oceania region, and information on HCRU for patients with SEA is limited in this setting. Moreover, country- and ethnicity-specific evidence comparing clinical characteristics of SEA with general asthmatics are needed to better characterise patients with this SA phenotype. The aim of this study was to evaluate prevalence of patients classified with SEA from the HealthStat primary care database in New Zealand, describe their characteristics, exacerbations, treatment patterns, HCRU costs (total and asthmarelated) in relation to non-SEA asthmatics (a patient cohort excluding those classified with SEA).

\section{Methods}

\section{Study design}

This was a retrospective observational cohort study of asthmatic patients enrolled in the HealthStat primary care database, which contains electronic medical records of patients registered with a general practice (GP) - over $98 \%$ of the New Zealand population. Since 2005 the HealthStat database has included data for 500,000 patients from 102 randomly selected, geographically representative GP clinics. ${ }^{15}$ All data related to consultations, prescriptions, laboratory results, clinical measurements and diagnostic coding for each patient in each practice are uploaded into the database on a weekly basis. Data from the National Minimum Dataset (NMDS; ICD codes) are linked to primary care data in HealthStat using patient unique health identifiers (National Health Index [NHI] codes) to describe utilization of emergency department, outpatient hospital visits and inpatient hospital admissions. Data from 20112012 were collated for this study.

\section{Study population}

Patients were identified using asthma diagnosis and hospital codes from the HealthStat general practice database (using READ codes) and the NMDS (using ICD codes). Participants were eligible for this study if they had any recorded diagnosis of asthma with no concurrent COPD in their clinical record and had eosinophil laboratory data. The index date was set as 31 December, 2011, for patients meeting all the following criteria:

1. $\geq 1$ diagnostic code for asthma (READ codes: H33*, 663V*; ICD code: J45, in any of up to 15 diagnoses made in a hospital in the last 3 years) before index date OR $\geq 2$ ICS-containing prescriptions during the calendar year in 2011 (in case asthma diagnosis codes recorded during earlier years in the medical records were not re-recorded for 2011);

2. Age $\geq 18$ on index date;

3. $>1$ prescription for an ICS-containing product (+/- LABA) between 1 January and 31 December 2011;

4. Patients must have been enrolled with the practice as a registered, funded patient for 1 year prior to and following the index date (followed until 31 December 2012);

5. No recorded diagnosis prior to the index date at any time in the medical record for chronic obstructive pulmonary disease, cystic fibrosis, lung cancer, bronchiectasis, or fibrotic lung disease.

A 1-year baseline period prior to and not including the index date was used to classify patients with SEA using the criteria below, and a 12-month observation period following the index date (from 1 January 2012 to 31 December 2012) was used to capture exacerbation history, treatment, medical and HCRU, and HCRU costs (Supplementary Figure 1).

\section{SEA patients}

Asthma patients with no concomitant COPD diagnosis identified during the baseline period were considered to have SEA if they met all following criteria, in alignment with phenotypic characteristics identified and used in previously published literature $[15,16]$ during the baseline period prior to index date:

1. GINA Step 4 or 5 treatment (Step 4: Medium-/high-dose ICS/LABA; Step 5: Refer for add-on treatment) [17] with ICS exposure (prescription record) above medium dose during the 12-month baseline period;

2. A history of $\geq 2$ exacerbations (defined by OCS burst or a GP visit with an asthma ICD code) during the baseline period;

3. Blood eosinophil count $(\mathrm{BEC}) \geq 300$ cells $/ \mu \mathrm{l}$ recorded at any time in the past 12 -months or $\geq 150$ cells $/ \mu 1$ in the last 6 weeks prior to the index date (Note: Local experts were consulted on the maximum window period prior to baseline as reasonable for patient recruitment and confirmed 6 weeks as appropriate).

All data for the present study other than the eosinophil counts had been collated for a previous asthma study in the HealthStat patient population [18]. For the present study, the general practices included in the HealthStat database were re-contacted to obtain permission to receive routine laboratory data with complete blood count test results for all patients. Prior to contacting the general 
practices, approval was obtained from the Health and Disability Ethics Committee in New Zealand. Eosinophil counts were extracted from the laboratory tests available in electronic format in the general practices and downloaded into the HealthStat database.

\section{Non-SEA patients}

Non-SEA patients were classified as asthma patients with no concomitant COPD diagnosis identified during the baseline period who did not meet the criteria described above for SEA. This category also included only asthma patients with available eosinophil laboratory data.

\section{More severe subgroup}

An additional subgroup of scientific interest was defined to further explore the SEA population amongst asthma patients with/without concurrent diagnosis of COPD using the following criteria, which were more stringent than those used to define the SEA population:

1. A history of $\geq 4$ exacerbations (OCS burst) during the 12month baseline period;

2. Treated with high-dose ICS (equivalent to $1000 \mathrm{mcg}$ fluticasone propionate per day) and a long acting $\beta-2$ agonist (LABA);

3. BEC of [19]:

a. $>500$ cells $/ \mu \mathrm{L}$ in the 6 weeks prior to index date, $\mathrm{OR}$

b. $>300$ cells $/ \mu \mathrm{L}$ in the 12 -month baseline period

\section{Description of patients (baseline period)}

Demographics and clinical characteristics: Age, gender, ethnicity, comorbidities, height and weight, smoking history, complete white blood cell count, blood neutrophil count and antibiotic prescription data were obtained from the HealthStat database for the baseline period.

Medication usage: Mean prescriptions and proportions of patients prescribed $\geq 1$ of the following asthma medications over the 12-month baseline period were described: LAMA (long-acting muscarinic antagonist), LABA, ICS, ICS+LABA, ICS+LAMA, triple therapy (LABA/LAMA/ICS), short-acting bronchodilator (SABD). Based on medical record data, treatments were calculated as mutually exclusive categories. Antibiotic prescription was measured to assess presence of active infection at time of BEC. Other asthma medications not listed above (including leukotriene receptor antagonist, systematic beta agonist, omalizumab) were not prescribed in the participating general practices during the study period. Medication coverage (as percentage of days covered by prescription for asthma treatment) was determined assuming 90 days of coverage for each prescription.

Asthma control: In this study, asthma control was defined using the number of exacerbations experienced by a patient during the 12 -month baseline period. Patients experiencing $\geq 2$ exacerbations were considered to have uncontrolled asthma [20].

\section{Outcome definitions (follow up period)}

Medication usage: Mean prescriptions and proportions of patients prescribed $\geq 1$ asthma medications over the 12-month follow up period was described as indicated for the 12-month baseline period.

Asthma control: Asthma control during the 12-month follow up period was defined as indicated for the 12-month baseline period. Exacerbations were defined as having $\geq 1$ of the following over the 12-month follow up period in 2012: 1) GP, outpatient hospital, emergency department visit, or hospital admission related to an asthma diagnosis/discharge code; 2) recorded prescription of any OCS.
$H C R U$ : For the 12-month follow up period during 2012, patients were assessed for total (all-cause) and asthma-related healthcare encounters (GP visits), outpatient hospital visits, emergency department visits and hospitalization days, using the supplemental healthcare utilisation data from the 2012 NMDS.

HCRU costs: Using the NMDS, the total and asthma-related costs associated with GP visits, hospitalizations, emergency department visits, and outpatient hospital visits were summarized for SEA patients and non-SEA asthma patients during 2012.

\section{Statistical analysis}

The proportion of patients meeting criteria for SEA during the baseline period was calculated. Patient demographics, clinical characteristics, treatment patterns, and HCRU for SEA and nonSEA patients were summarized with arithmetic mean, standard deviation (SD) for continuous variables and as number and proportion for categorical variables - this study was descriptive, and no statistical comparison was made between the identified SEA and non-SEA patient groups.

Based on medical record data, treatments were classified as mutually exclusive categories. The number of days' supply was unavailable; it was assumed each medication prescription was of 90-day duration, which is typical for maintenance medications in New Zealand [21].

Asthma-related costs were considered as costs associated with an asthma primary diagnosis code. Annual cost data were derived from the NMDS and were obtained by multiplying the cost weight for each service (a supplied file from the NMDS extraction) by the base unit cost for the service in 2012. The reported cost (New Zealand dollars, NZD) is the average amount per person during 2012.

Missing data were detailed as necessary and no imputation was conducted. No additional data cleaning was performed on the data collated and routinely loaded into the HealthStat database. No sample size or power/precision calculations were performed.

\section{Results}

\section{Patient populations}

Among the total HealthStat database population for the 2011 baseline period, eosinophil laboratory measurements were available for 3,276 of all asthma patients and out of these 160 (4.9\%) patients met the three additional criteria for SEA, i.e. treated with high dose ICS + LABA, $\geq 2$ exacerbations in the baseline period and $\mathrm{BEC} \geq 300$ cells/ $\mu 1$ during the entire 12 -month baseline period or $\geq 150$ cells $/ \mu \mathrm{l}$ in the 6 weeks prior to the index date (Figure 1).

\section{Demographics, clinical characteristics and asthma control}

Patients were more likely to be female in both groups. Compared with non-SEA patients, SEA patients were older, more likely to be Māori, and more likely to have smoked. Greater proportions of SEA patients than non-SEA patients had comorbidities, a higher BMI, and greater neutrophil counts (Table 1). Out of the 3,276 asthma patients with eosinophil data, 70 (2.1\%) experienced $\geq 4$ exacerbations in the 12-month baseline period (Supplementary Table 1); when examined by ethnicity, incidence of exacerbations of the same frequency were highest for Māori patients (46/174, 26.4\%; Supplementary Figure 2A).

In the total study population, $4.8 \%$ of patients who also belonged to the more severe subgroup received high-dose ICS + LABA in the 12-month baseline period, and of the different ethnicities, more Māori patients were receiving this treatment (41/156, 26.3\%; Supplementary Figure 2A). The more severe sub- 
group comprised of patients with BEC of $>300$ cells $/ \mu 1$ in the 12month baseline period or BEC of $>500$ cells $/ \mu l$ in the 6 weeks prior to index date; patients with the lower eosinophil count and longer validity window for eosinophils made up $1.9 \%$ of the total population. This number dropped to $0.1 \%$ when the higher threshold was applied. When examined by ethnicity, Māori patients constituted the highest percentage of patients for both BECs (Supplementary Figure 2B).

In the 12-month follow up period, $56.2 \%$ of SEA patients had uncontrolled asthma compared with $11.2 \%$ of non-SEA patients; the mean number of exacerbations for SEA patients was four times as many as the non-SEA group and around a third experienced $\geq 4$ exacerbations compared with $3.1 \%$ of non-SEA patients (Table 2 ).

\section{Treatment patterns}

For SEA patients, percentage of days covered by prescriptions for asthma medications was over twice that for non-SEA patients (45.2\% vs 20.6\%, respectively); SEA patients received greater mean numbers of prescriptions for the majority of the assessed respiratory medications including 4.8 times as many for OCS, over twice as many for combined ICS + LABA and single LAMA (Table 3). In the 12-month follow up period, the number of non-

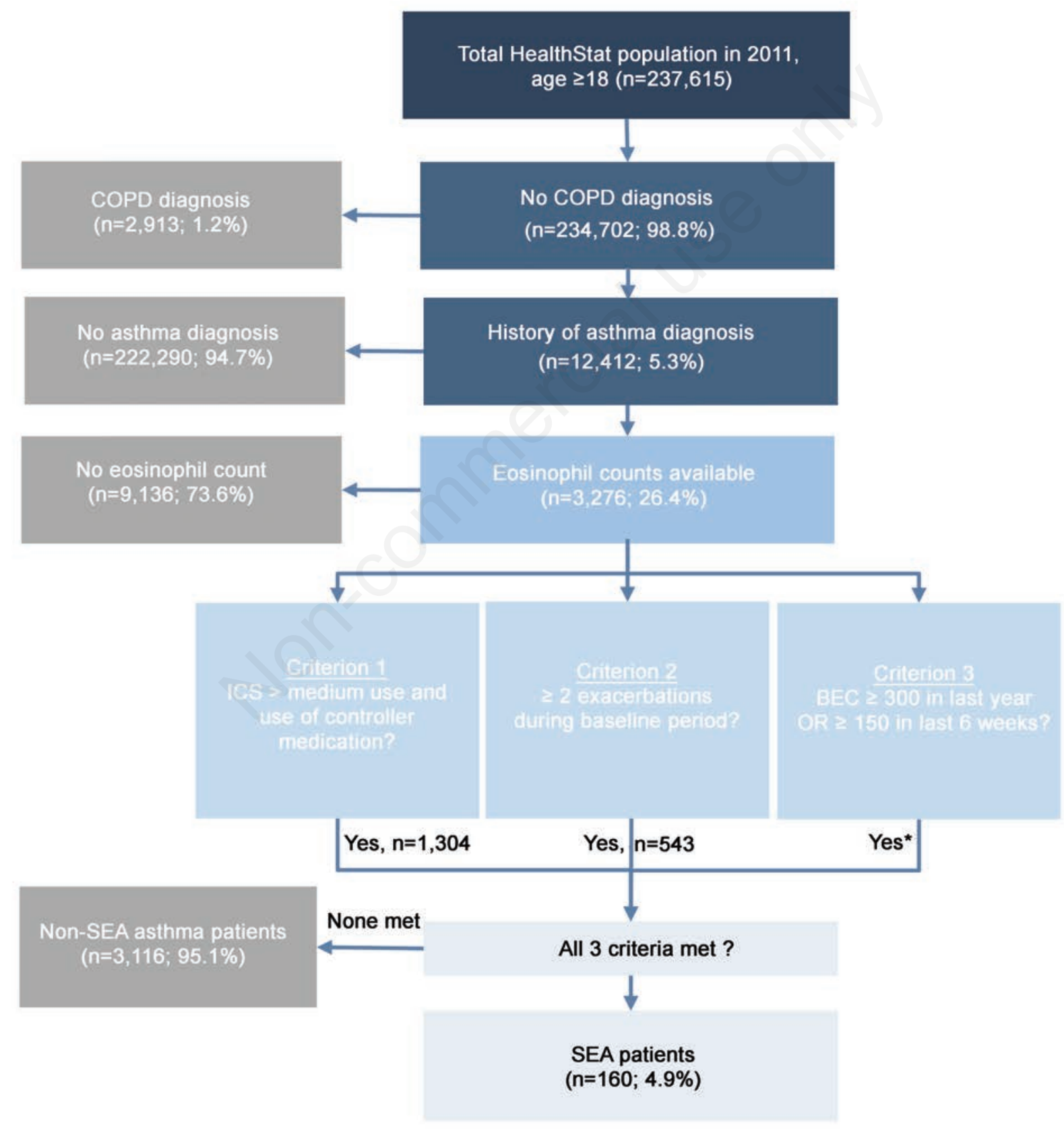

Figure 1. Patient flow diagram. ${ }^{*}=1,619$ with eosinophil count of $\geq 300$ in the last year and $n=429$ with eosinophil count of $\geq 150$ in last 6 weeks. BEC, blood eosinophil count; ICS, inhaled corticosteroids; SEA, severe eosinophilic asthma. 
SEA patients not receiving treatment was approximately six times greater than SEA patients (16.2\% vs $2.5 \%$, respectively), and fewer SEA patients than non-SEA patients were receiving ICS alone. Proportions of patients receiving no treatment in the follow up period was greater for both non-SEA and SEA patients than the baseline period (Table 3, Supplementary Table 2).

\section{Healthcare resource utilisation and costs}

Patients classified with SEA utilized up to 1.5 -fold more healthcare resources than non-SEA patients in the 12-month follow up period - this was for all services assessed, but principally for pri- mary care (GP visits: $20.7 \pm 14.1$ vs $13.0 \pm 10.3$, respectively; Table 4). Compared with those hospitalized non-SEA asthma patients, hospitalized SEA patients incurred over three times higher mean annual costs for asthma-related HCRU (282.7 NZD vs 78.1 NZD, respectively), and higher mean costs for total HCRU (Figure 2 A,B).

\section{Disease and treatment evolution}

Asthma control in this study, as defined by exacerbation number, remained stable over time in the non-SEA patient group (controlled asthma: $87.7 \%$ in the baseline period and $88.8 \%$ in the

Table 1. Baseline demographics and clinical characteristics.

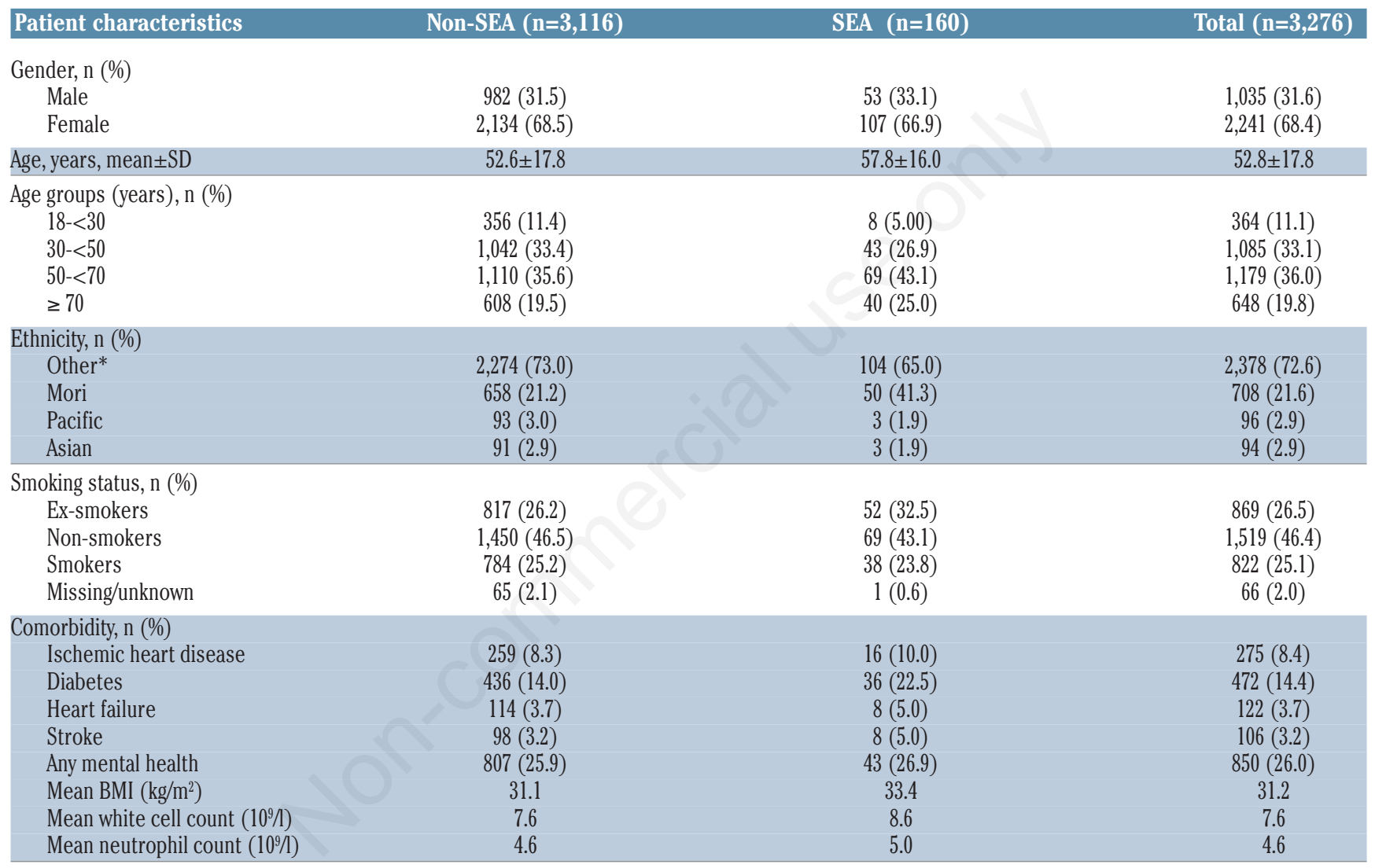

SEA, severe eosinophilic asthma; SD, standard deviation; *New Zealand European, British and Irish, Australian, Middle Eastern, African; BMI, body mass index.

Table 2. Asthma control and exacerbations in the 12-month follow up period.

\begin{tabular}{lccc} 
Items & Non-SEA $(\mathbf{n = 3 1 1 6 )}$ & Total (n=3276) \\
History of exacerbations, $n(\%)$ & & & \\
$\quad$ None & $2,127(68.3)$ & $37(23.1)$ & $2,164(66.1)$ \\
$1-2$ & $665(21.3)$ & $56(35.0)$ & $721(22.0)$ \\
3 & $74(2.4)$ & $19(11.9)$ & $93(2.9)$ \\
$\quad 4$ & $95(3.1)$ & $46(28.8)$ & $141(4.3)$ \\
$\quad$ Missing & $155(5.0)$ & $2(1.3)$ & $157(4.8)$ \\
Patients with controlled asthma* & $2,767(88.8)$ & $70(43.8)$ & $2,837(86.6)$ \\
Patients with uncontrolled asthma* & $349(11.2)$ & $90(56.2)$ & $439(13.4)$ \\
No. of exacerbations mean \pm SD & $0.6 \pm 1.3$ & $2.4 \pm 2.2$ & $0.6 \pm 1.4$ \\
No. of GP visits, mean \pm SD & $13.0 \pm 10.3$ & $20.7 \pm 14.1$ & $13.3 \pm 10.7$ \\
\hline
\end{tabular}

SEA, severe eosinophilic asthma; * patients experiencing $\geq 2$ exacerbations in the follow up period were considered to have uncontrolled asthma; patients with <2 exacerbations were considered to have controlled asthma; SD, standard deviation; GP, general practice. 
follow up period) and increased in the SEA patient group (controlled asthma: $0 \%$ in the baseline period and $43.8 \%$ in the follow up period). Mean exacerbation number for non-SEA patients remained stable over time $(0.6 \pm 1.3$ in the baseline period and $0.6 \pm 1.3$ in the follow up period) and decreased for SEA patients
(3.8 \pm 2.2 in the baseline period and $2.4 \pm 2.2$ in the follow up period; Table 2, Supplementary Table 1). Percentage of days covered by prescriptions for asthma medications increased over time for nonSEA patients with doubled improvement for SEA patients, while mean numbers of prescriptions increased in the follow up period vs
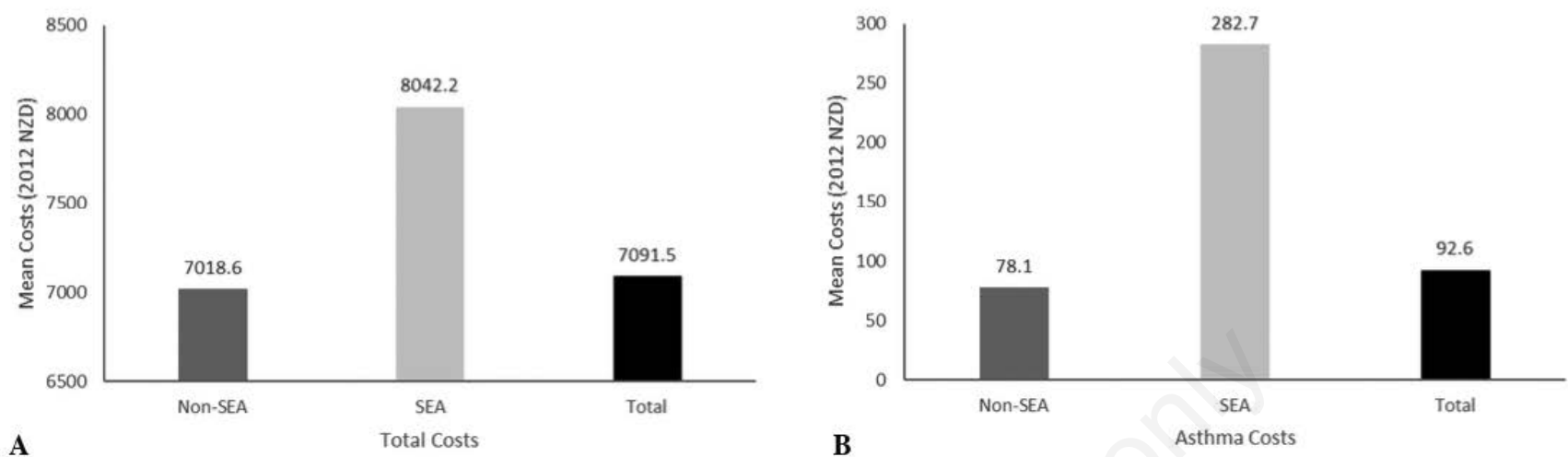

Figure 2. Total and asthma-related healthcare resource utilization costs for asthma patients hospitalized during the 12-month follow up period. A) Total healthcare resource utilization costs. B) Asthma-related healthcare resource utilization costs. NZD, New Zealand dollars; SEA, severe eosinophilic asthma.

Table 3. Respiratory medication usage during the 12-month follow up period.

\begin{tabular}{|c|c|c|c|}
\hline Items & Non-SEA $(n=3,116)$ & SEA $(n=160)$ & Total $(n=3,276)$ \\
\hline $\begin{array}{l}\text { Percentage of days covered* by prescription for asthma treatment, } \% \\
\text { No. of prescriptions, mean }\end{array}$ & 37.5 & 90.8 & - \\
\hline Any oral corticosteroids & 0.5 & 2.4 & 0.6 \\
\hline Any single LABA & 0.2 & 0.0 & 0.2 \\
\hline Any single ICS & 1.1 & 0.1 & 1.1 \\
\hline Any single LAMA & 0.1 & 0.2 & 0.1 \\
\hline SABD & 1.9 & 3.5 & 2.0 \\
\hline Combined ICS + LABA & 1.3 & 3.6 & 1.5 \\
\hline Antibiotics & 0.2 & 0.6 & 0.3 \\
\hline Total & 5.4 & 10.4 & 5.6 \\
\hline \multicolumn{4}{|l|}{ Treatment use, \% (mutually exclusive categories) } \\
\hline 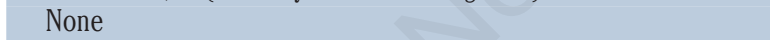 & 16.2 & 2.5 & 15.6 \\
\hline ICS alone & 32.7 & 1.3 & 31.2 \\
\hline ICS/LABA & 41.6 & 85.6 & 43.8 \\
\hline ICS/LAMA & 0.2 & 0 & 0.2 \\
\hline LABA & 0.4 & 0.6 & 0.4 \\
\hline ICS/LABA/LAMA & 1.7 & 4.4 & 1.8 \\
\hline LAMA & 0 & 0 & 0 \\
\hline SABD alone & 7.1 & 5.6 & 7.1 \\
\hline
\end{tabular}

SEA, severe eosinophilic asthma; *assuming 90 days of coverage for each prescription. ICS, inhaled corticosteroid; LABA, long-acting -agonist; LAMA, long-acting muscarinic antagonist; SABD, short-acting bronchodilator.

Table 4. Healthcare resource utilization during the 12-month follow up period.

\begin{tabular}{lccc}
\hline Items, mean \pm SD & Non-SEA $(\mathbf{n = 3 , 1 1 6 )}$ & SEA $(\mathbf{n = 1 6 0 )}$ & Total $(\mathbf{n = 3 , 2 7 6 )}$ \\
GP visits & $13.0 \pm 10.3$ & $20.7 \pm 14.1$ & $13.3 \pm 10.7$ \\
Emergency department visits & $0.4 \pm 1.2$ & $0.5 \pm 1.0$ & $0.4 \pm 1.2$ \\
\hline Outpatient visits & $2.6 \pm 11.9$ & $3.8 \pm 7.9$ & $2.7 \pm 11.7$ \\
Total hospitalized days & $0.9 \pm 5.2$ & $1.3 \pm 3.9$ & $1.0 \pm 5.1$ \\
\hline Asthma-related hospitalized days & $0.0 \pm 0.3$ & $0.1 \pm 0.6$ & $0.0 \pm 0.33$ \\
\hline
\end{tabular}

SEA, severe eosinophilic asthma; SD, standard deviation; GP, general practice. 
the baseline period for both patient groups. Proportions of patients receiving no treatment in the follow up period was greater for both non-SEA and SEA patients than the baseline period (Table 3, Supplementary Table 2).

\section{Discussion}

The aim of this retrospective cohort study was to characterize the epidemiology, clinical characteristics, HCRU, and costs for patients classified with SEA in New Zealand, as data describing the SEA phenotype of SA are especially lacking in Asia Pacific/Oceania.

We found greater proportions of patients classified with SEA had a history of smoking, comorbidities, and on average were older than non-SEA patients (58 vs 53 years). That the SEA patient group was older reflects findings from a UK study [20], and contrasts with a study in Taiwan in which the mean age of SEA and general asthma groups were similar [21]. In this New Zealand study, a greater proportion of patients with SEA had a higher BMI compared with non-SEA patients. This aligns with study results from the UK where more patients with severe uncontrolled eosinophilic asthma were obese compared with all asthma patients [20]. The prevalence of patients classified with SEA among all asthma only patients (i.e., no concurrent COPD diagnosis) identified from the HealthStat database in New Zealand in this study was $4.9 \%$ (defined using the criteria in our study of: GINA Step 4 or 5 treatment 17 with an ICS exposure above medium dose during the baseline period; $\geq 2$ exacerbations during the baseline period; and $\mathrm{BEC} \geq 300$ cells $/ \mu \mathrm{l}$ recorded at any time in the past 12 -months or $\geq 150$ cells/ $\mu \mathrm{l}$ in the 6 weeks prior to the index date). This is similar to previous estimates reported in other countries [22-24], and the same as that recently identified from a similar study in Taiwan [21]. Asthma prevalence in children and adults in New Zealand has been shown to be greater in the Māori population compared with non-Māori [25]. Other literature also indicates a higher asthma prevalence and health burden among Māori than non-Māori New Zealanders [26-29]. Known clinical features associated with severe exacerbations also include Māori ethnicity [30]. We found that both non-SEA and SEA patient groups consisted of greater proportions of Māori patients than Pacific and Asian patients, in support of a disproportionate prevalence of asthma and SEA in this population. Importantly, our real-world study is the first indicating a higher prevalence of SEA in the Māori population.

The underlying cause of the disproportionate prevalence of asthma and SEA in the Māori population remains unknown, although may be due to genetic and/or environmental differences or risk factors, for example higher proportions of Māori live in areas scored as more highly deprived [31], which is important because asthma severity has been shown to be linked to factors associated with poverty [32]. Additionally, there is a known socioeconomic gradient in asthma hospitalizations in New Zealand, with the most socioeconomically deprived areas having higher asthma hospitalization rates, and accordingly Māori have higher hospitalization rates than other ethnicities in New Zealand [9,25] Despite this, however, Māori patients with asthma are less likely to receive ICS prescriptions [30]. Therefore, these more deprived patients may be under-treated, which may lead to worse disease outcomes in this population.

\section{Subgroup results}

In this study a subgroup of SEA patients was also analyzed for representation by ethnicity. This subgroup was defined by more stringent criteria to identify those patients in the total study popu- lation with the greatest exacerbation history, who were receiving high-dose ICS+LABA, and had the highest BECs. Of the ethnicities analyzed in this study, Māori patients experienced exacerbations more frequently than Pacific and Asian patients. This indicates these Māori patients with high BECs have poorly controlled disease and a high disease burden despite receiving high-dose ICS treatment and therefore have a high unmet need for more effective therapy.

\section{Disease and treatment evolution}

Of note, we found that asthma control in non-SEA classified patients remained stable between the baseline and follow up periods, whereas control increased in the SEA patient group. Additionally, more patients in both SEA and non-SEA groups received no treatment in the follow up compared with the baseline period. Together these data could indicate: a change in these patients' asthma condition over time such that medication was no longer required; stabilization of asthma severity over a longer term leading to improved control; receipt of medications from community pharmacies that were not included in the HealthStat database; a change in prescription of asthma medication, or utilization of advice for disease control, led to better symptom relief; or potentially could reflect a reduction in primary care visits - i.e. in compliance, in which case medications may still have been required but not prescribed and therefore the data would be absent from the HealthStat database. The number of GP visits for both populations decreased in the follow up vs the baseline period, however as there was also an increase in proportion of patients who were classified as having controlled asthma it is more probable that patients experienced a change in their condition or better symptom control due to changes in medication prescription.

\section{Healthcare resource utilization}

During the follow up period, although SEA patients had approximately twice as many respiratory treatment prescriptions than non-SEA patients - as could be expected for patients with more severe asthma - the percentage of days of medication coverage for SEA patients was high $(>90 \%)$. The most common treatment for SEA patients in the follow up period other than ICS/LABA and SABD was OCS, in contrast to treatments such as Theophylline, leukotriene receptor antagonists and fixed combinations of SABA+short-acting muscarinic antagonists (SAMA) reported for patients classified with SEA in Taiwan [21].

Healthcare utilization was higher for patients classified with SEA than non-SEA patients in the follow up period: SEA patients incurred higher mean total costs, and 3.6 times higher asthmarelated mean annual costs, compared with non-SEA asthma patients. Relative to other studies, the difference in asthma-related costs for patients classified with SEA compared with non-SEA patients in New Zealand was greater than that in Taiwan [21], and more similar to that in the UK (cost ratio of 3.9 [95\% CI 3.7 to 4.1]) [20].

\section{Limitations}

This study had several limitations. Firstly, there may have been differences in demographic and clinical characteristics between participants with or without follow up visits after the index date, which may have affected the analysis of medication usage and exacerbation frequency in the follow up period. For example, patients without the full year of follow up may have had less severe asthma and therefore less need for medical care. Additionally, patients that were not continuously enrolled with a practice may have been more economically disadvantaged and had more severe asthma. Primary care diagnosis and prescribing data were derived from the HealthStat database from patients who were registered 
with a primary care provider at the start and end of the study. Therefore, those patients without the full year of follow up data may have been lost to the database if, for example, they were transient, i.e. they registered with a different practice during the study. These patients would have been underrepresented in the study population. Secondly, results from this study may have been underestimated in several ways. Exacerbations and health burden of SEA patients may have been underestimated in this study as a more restrictive definition for exacerbations was used at baseline (baseline: OCS burst or GP visit with an asthma ICD code). In the follow up period however, the definition was expanded to include OCS burst, GP, outpatient hospital and emergency department visit or hospital admission related to an asthma diagnosis/discharge code, and recorded OCS prescription. The definition of asthma control also usually encompasses lung function and symptoms criteria, therefore asthma control may have been underestimated in our study, as only exacerbations were used in our definition. Thirdly, the definition for SEA in this study included an ICS daily dose during the baseline period that was higher than the cut off for medium dose, in-line with GINA recommendation in defining patients with severe asthma [17]. Depending on the number of puffs per day and considering the possibility of non-compliance, the dose may not have met the threshold for high dose. However, most patients were expected to meet the high ICS dose threshold. Fourthly, serological tests conducted in the hospital may not have been reported back to primary care physicians and were missed in our analysis. Fifthly, HealthStat only captures diagnosed diseases in the primary care setting. Patients who were symptomatic for COPD, but had not yet received a COPD diagnosis (potentially milder patients) may have been included in this study. Another limitation is the descriptive nature of the study, whereby no statistical testing for differences between the identified SEA and non-SEA patient groups was conducted.

\section{Conclusions}

Asthma patients classified with SEA in New Zealand, approximately $40 \%$ of whom were Māori, had greater healthcare utilization, medication usage, total and asthma-related costs compared with non-SEA patients. Māori patients were also more highly represented in a subgroup of SEA patients classified by more stringent exacerbation and treatment criteria than other ethnicities, indicating more severe disease and a high unmet treatment need in these patients. Our data are expected to be highly generalizable to the New Zealand population given the representation of $\sim 10 \%$ of the population in the HealthStat database.

\section{Acknowledgements}

Initial drafts of the manuscript were developed by Ruth Le Fevre, PhD, Costello Medical, Singapore, and was funded by GSK. Approval draft development and finalisation of the manuscript were completed by the authors.

$\begin{array}{ll}\text { Abbreviations } \\ \text { BEC: } \quad \text { Blood eosinophil count } \\ \text { BMI: } \quad \text { Body mass index } \\ \text { COPD: } & \text { Chronic obstructive lung disease } \\ \text { GINA: } & \text { Global Initiative for Asthma } \\ \text { GP: } & \text { General practice } \\ \text { HCRU: } & \text { Healthcare resource utilization }\end{array}$

ICS: Inhaled glucocorticoids

LABA: Long acting $\beta-2$ agonist

LAMA: Long-acting muscarinic antagonist

NHI: National health index

NMDS: National minimum dataset

NZD: New Zealand dollars

OCS: Oral corticosteroid

SA: $\quad$ Severe asthma

SABD: Short-acting bronchodilator

SAMA: Short-acting muscarinic antagonists

SD: $\quad$ Standard deviation

SEA: Severe eosinophilic asthma

\section{References}

1. Green RH, Brightling CE, McKenna S, Hargadon B, Parker D, Bradding $\mathrm{P}$, et al. Asthma exacerbations and sputum eosinophil counts: a randomised controlled trial. Lancet 2002;360:171521.

2. Chung KF, Wenzel SE, Brozek JL, Bush A, Castro M, Sterk PJ, et al. International ERS/ATS guidelines on definition, evaluation and treatment of severe asthma. Eur Respir J 2014;43:343373.

3. Calhoun WJ, Haselkorn T, Mink DR, Miller DP, Dorenbaum A, Zeiger RS. Clinical burden and predictors of asthma exacerbations in patients on guideline-based steps 4-6 asthma therapy in the TENOR cohort. J Allergy Clin Immunol Pract 2014;2:193-200.

4. Serra-Batlles J, Plaza V, Morejon E, Comella A, Brugués J. Costs of asthma according to the degree of severity. Eur Respir J 1998;12:1322-6.

5. Dean BB, Calimlim BC, Sacco P, Aguilar D, Maykut R, Tinkelman D. Uncontrolled asthma: assessing quality of life and productivity of children and their caregivers using a crosssectional Internet-based survey. Health Qual Life Outcomes 2010;8:96.

6. Fleming L, Murray C, Bansal AT, Hashimoto S, Bisgaard H, Bush A, et al. The burden of severe asthma in childhood and adolescence: results from the paediatric U-BIOPRED cohorts. Eur Respir J 2015;46:1322.

7. Shaw DE, Sousa AR, Fowler SJ, Fleming LJ, Roberts G, Corfield J, et al. Clinical and inflammatory characteristics of the European U-BIOPRED adult severe asthma cohort. Eur Respir J 2015;46:1308-1321.

8. Bahadori K, Doyle-Waters MM, Marra C, Lynd L, Alasaly K, Swiston J, et al. Economic burden of asthma: a systematic review. BMC Pulm Med 2009;9:24-24.

9. Barnard L, Baker M, Pierse N, Zhang J. The impact of respiratory disease in New Zealand: 2014 update. The Asthma Foundation 2015. Last accessed: February 2019. Available from: www.asthmafoundation. org.nz/research/the-impact-ofrespiratory-disease-in-new-zealand-2014-update

10. Lai CK, Beasley R, Crane J, Foliaki S, Shah J, Weiland S. Global variation in the prevalence and severity of asthma symptoms: phase three of the International Study of Asthma and Allergies in Childhood (ISAAC). Thorax 2009;64:476-83.

11. Crane J, Lewis S, Slater T, Rossland L, Robson B, D'Souza W, et al. The self reported prevalence of asthma symptoms amongst adult New Zealanders. N Z Med J 1994;107:417-21.

12. Ellison-Loschmann L, Pattemore PK, Asher MI, Clayton TO, Crane J, Ellwood P, et al. Ethnic differences in time trends in asthma prevalence in New Zealand: ISAAC Phases I and III. Int J Tuberc Lung Dis 2009;13:775-82.

13. Pattemore PK, Ellison-Loschmann L, Asher MI, Barry DM, 
Clayton TO, Crane J, et al. Asthma prevalence in European, Maori, and Pacific children in New Zealand: ISAAC study. Pediatr Pulmonol 2004;37:433-42.

14. Gillies TD, Tomlin AM, Dovey SM, Tilyard MW. Ethnic disparities in asthma treatment and outcomes in children aged under 15 years in New Zealand: analysis of national databases. Prim Care Respir J 2013;22:312-8.

15. Pavord ID, Korn S, Howarth P, Bleecker ER, Buhl R, Keene $\mathrm{ON}$, et al. Mepolizumab for severe eosinophilic asthma (DREAM): a multicentre, double-blind, placebo-controlled trial. Lancet 2012;380:651-9.

16. Ortega HG, Liu MC, Pavord ID, Brusselle GG, FitzGerald JM, Chetta A, et al. Mepolizumab treatment in patients with severe eosinophilic asthma. N Engl J Med 2014;371:1198-207.

17. Global Initiative for Asthma. Global Strategy for Asthma Management and Prevention. Last accessed: February 2019. Available from: https://www.ucalgary.ca/icancontrolasthma/ files/icancontrolasthma/wms-gina-2016-main-report-final.pdf

18. Shantakumar S, Yu-Fan H, Beale J, Gribben B. An observational study to characterize severe eosinophilic asthma in New Zealand. Eur Respir J 2018;52:PA1155.

19. Respiratory Subcommittee of the Pharmacology and Therapeutics Advisory Committee (PTAC). Mepolizumab for severe eosinophilic asthma - minutes of the 2017 PTAC meeting. Last accessed: October 2019. Available from: https:/www.pharmac.govt.nz/assets/ptac-respiratory-subcommittee-minutes-2017-08.pdf

20. Kerkhof M, Tran TN, Soriano JB, Golam S, Gibson D, Hillyer $\mathrm{EV}$, et al. Healthcare resource use and costs of severe, uncontrolled eosinophilic asthma in the UK general population. Thorax 2018;73:116-24.

21. Shantakumar S, Yu-Fan H, Li-Wen T, Tsu-Mei L, Yi-Hsing C. A retrospective observational study to characterize severe eosinophilic asthma in Taiwan. Eur Respir J 2018;52:PA3917.

22. Lang JE, Hossain J, Smith K, et al. Asthma severity, exacerbation risk, and controller treatment burden in underweight and obese children. J Asthma 2012;49:456-63.

23. Jarjour NN, Erzurum SC, Bleecker ER, Lima JJ. Severe asthma: lessons learned from the National Heart, Lung, and Blood Institute Severe Asthma Research Program. Am J Respir Crit
Care Med 2012;185:356-62.

24. de Carvalho-Pinto RM, Cukier A, Angelini L, Antonangelo L, Mauad T, Dolhnikoff M, et al. Clinical characteristics and possible phenotypes of an adult severe asthma population. Respir Med 2012;106:47-56.

25. Barnard L, Zhang J. The impact of respiratory disease in New Zealand: 2018 update. Last accessed: April 2019. Available from: https://s3-ap-southeast-2.amazonaws.com/assets.asthmafoundation.org.nz/images/NZ-Impact-Report-2018_ FINAL.pdf

26. New Zealand Ministry of Health. Tatau Kahukura Māori Health Chart Book 2010. Wellington: Ministry of Health, 2010.

27. Lewis S, Hales S, Slater T, Pearce N, Crane J, Beasley R. Geographical variation in the prevalence of asthma symptoms in New Zealand. N Z Med J 1997;110:286-9.

28. New Zealand Ministry of Health. Ngā mana hauora tūtohu: Health status indicators - Asthma. 2018. Last accessed: March 2019. Available from: https://www.health.govt.nz/ourwork/populations/maori-health/tatau-kahukura-maori-healthstatistics/nga-mana-hauora-tutohu-health-statusindicators/respiratory-disease.

29. Robson B, Harris R. Hauora: Māori Standards of Health IV. A study of the years 2000-2005. Wellington: Te Ropu Rangahau Hauora a Eru Pomare; 2007.

30. Beasley R, Hancox RJ, Harwood M, Perrin K, Poot B, Pilcher $\mathrm{J}$, et al. Asthma and Respiratory Foundation NZ adult asthma guidelines: a quick reference guide. N Z Med J 2016;129:83102.

31. New Zealand Ministry of Health. Neighbourhood deprivation. 2013. Last accessed: April 2019. Available from: https://www.health.govt.nz/our-work/populations/maorihealth/tatau-kahukura-maori-health-statistics/nga-awe-o-tehauora-socioeconomic-determinants-health/neighbourhooddeprivation

32. Poyser MA, Nelson H, Ehrlich RI, Bateman ED, Parnell S, Puterman A, et al. Socioeconomic deprivation and asthma prevalence and severity in young adolescents. Eur Respir J 2002;19:892.

Received for publication: 6 April 2020. Accepted for publication: 17 June 2020.

This work is licensed under a Creative Commons Attribution-NonCommercial 4.0 International License (CC BY-NC 4.0).

(C) Copyright: the Author(s), 2020

Licensee PAGEPress, Italy

Multidisciplinary Respiratory Medicine 2020; 15:662

doi:10.4081/mrm.2020.662 